\title{
Complex Hydrates in the Lower lonosphere
}

ON the basis of a model proposed by Nicolet and Aikin (J. Geophys. Res., 65, 1469; 1960) it was expected that under normal conditions $\mathrm{NO}^{+}$and $\mathrm{O}_{2}{ }^{+}$would be the dominant ions in the $\mathrm{D}$ region of the ionosphere, extending from about 60 to $95 \mathrm{~km}$. Measurements with a rocket-borne mass-spectrometer by Narcisi and Bailey $(J$. Geophys. Res., 70, 3687 ; 1965) of the Air Force Cambridge Research Laboratories confirmed that these were the major ions above about $83 \mathrm{~km}$, with metallic ions also being present. But they reported that at lower heights the predominant ions were $\mathrm{H}_{3} \mathrm{O}^{+}$and $\mathrm{H}_{5} \mathrm{O}_{2}{ }^{+}$. For several years there was some doubt whether these were ambient ions or the products of rocket contamination. The reproducibility of the observations during rocket ascent and descent on this and several subsequent flights have, however, confirmed their ambient nature. In the meantime, similar mass-spectrometry observations have been made by groups at the Max-Planck Institute for Nuclear Physics and the Goddard Space Flight Center.

A significant feature of all these observations is that under normal ionospheric conditions the water cluster ions disappear at a sharply defined level between 82 and $85 \mathrm{~km}$ above which $\mathrm{NO}^{+}$and $\mathrm{O}_{2}{ }^{+}$are dominant. This sudden change in ion composition is thought to be associated with the marked gradient in electron concentration often observed in the same height range (Reid, J. Geophys. Res., 75, 2551; 1970). The notion is that the change from the water cluster ions to $\mathrm{NO}^{+}$and $\mathrm{O}_{2}{ }^{+}$is accompanied by a decrease in electron loss for, as Professor Biondi and his associates at the University of Pittsburgh have shown, the recombination with electrons of such cluster ions is more rapid than that of $\mathrm{NO}^{+}$or $\mathrm{O}_{2}{ }^{+}$.

In this issue of Nature (page 215) Johannesen et al. describe a study of this transition region based on rocket observations of positive-ion composition, electron concentration, total positive- and negative-ion concentrations and energetic precipitated particles. The rocket was launched about an hour after noon during August 1971 at Andoya, on the east coast of Norway, the intention being to study conditions during the cold summer mesosphere ; measurements of the intensity of solar Lyman- $\alpha$ radiation as a function of height made on the same rocket were interpreted in terms of absorption by molecular oxygen to yield the height variation of atmospheric temperature. A particular feature of the mass-spectrometry experimenta was the relatively large mass range covered, 10-140 a.m.u., care having been taken to ensure sufficient sensitivity at the higher masses.

The observations indicate that water cluster ions, $\mathrm{H}^{+}\left(\mathrm{H}_{2} \mathrm{O}\right)_{n}$, dominated the ion composition below $84 \mathrm{~km}$ on both ascent and descent. Furthermore, cluster ions of higher order mass were seen to increase with height until an abrupt change occurred between 84.5 and $86 \mathrm{~km}$, at which height $\mathrm{NO}^{+}, \mathrm{O}_{2}{ }^{+}$and metal ions became predominant. The observation of the higher order cluster ions $\mathrm{H}^{+}\left(\mathrm{H}_{2} \mathrm{O}\right)_{3}, \mathrm{H}^{+}\left(\mathrm{H}_{2} \mathrm{O}\right)_{4}, \mathrm{H}^{+}\left(\mathrm{H}_{2} \mathrm{O}\right)_{5}$ and $\mathrm{H}^{+}\left(\mathrm{H}_{2} \mathrm{O}\right)_{6}$ is an important aspect of the results because it has been considered that perhaps these are the truly ambient ions, their fragmentation during the rocket sampling process giving rise to the lower order ions which are usually recorded. It is, perhaps, of interest to enquire whether the low mesospheric temperatures existing at the time of the flight render the weakly-bound cluster ions more stable.

During the period of the rocket flight the production of ion pairs was dominated by particle precipitation. The production rate showed little change in the height range in which the composition change was observed but the electron concentration and positive-ion concentration each showed an order of magnitude increase in this same height range. These results taken together indicate a sudden drop in the effective recombination rate where the cluster ions disappear, as expected from previous studies. But the more continuous measurements of electron concentration and positive-ion concentration show that this drop occurs in a height range of only 200 $300 \mathrm{~m}$, implying that the change in ion composition also occurs in such a small range of height.

The formation of these water cluster ions from ions produced during or following the primary ionization processes poses a major problem in the D-region. A rapid sequence of reactions beginning with $\mathrm{O}_{2}{ }^{+}$has been found from laboratory measurements but with a recent revision in the production rate of $\mathrm{O}_{2}{ }^{+}$this sequence is now thought to be inadequate below $75 \mathrm{~km}$ (Ferguson, Rev. Geophys., 9, $997 ;$ 1971). It is expected that a much more rapid production of $\mathrm{NO}^{+}$occurs, and considerable attention is being given to the possible role of this ion as a precursor of the cluster ions. The degree of hydration of $\mathrm{NO}^{+}$ions is a chief consideration in these studies and the observation by Johannesen et al. of ion masses attributed to the first and second hydrates will be noted with interest. The height variation of atmospheric temperature presented by Johannesen et al. shows no evidence of a discontinuity in the height range in which the water cluster ions disappear. It seems likely that their disappearance is associated with the onset of a reaction which interrupts the formation of the cluster ions (Ferguson, loc. cit.) rather than a temperature effect.-L. T.

\section{Regge Theory and the ISR}

NEW measurements being carried out on the Intersecting Storage Rings (ISR) at CERN to try to determine the precise shape of the diffraction peak in proton-proton scattering at ultra-high energies seem to promise surprising and intriguing results. If the proton can be thought of as an object with some definite size $\lambda$, then, according to classical diffraction theory, one would expect the width, $\Gamma$, of the diffraction peak (measured not in angle but as a width in the square of the momentum transfer) to remain constant as one goes to higher and higher collision energies, with $\Gamma \propto 1 / \gamma^{2}$. On the other hand, perhaps the most startling result of Regge pole theory is the prediction that the diffraction peak should shrink with increasing collision energy. More precisely, one expects $\Gamma \propto 1 /\left(\alpha^{\prime} \log E\right)$. Here $\alpha^{\prime}$, the slope of the dominant Regge trajectory, is a fixed number, and $E$ is the total energy in the centre-of-mass. Interpreted in classical terms, this means that the size of the proton increases with energy. This radical picture appeared to be substantiated by experiments done at CERN and Brookhaven some years ago, using protons with laboratory energies from 15 to $30 \mathrm{GeV}$, corresponding to $E \approx 5.5$ to $7.7 \mathrm{GeV}$, and by 